\title{
Publication guidelines for patterning tools
}

\author{
Harry J. Levinson \\ $\mathrm{JM}^{3}$ Editor-in-Chief
}

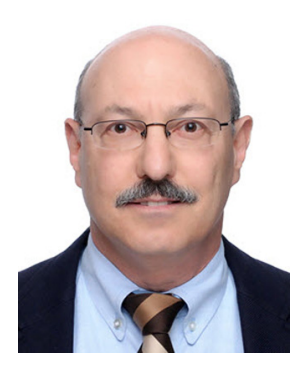

Previously, guidelines on the content of papers for $\mathrm{JM}^{3}$ were provided to authors and reviewers on the topics of lithographic materials [Vol. 19(3) of $\mathrm{JM}^{3}$ ] and metrology and masks [Vol. 20(2)], for achieving a balance between the inclusion of key data in published papers and the protection of crucial proprietary information. In this editorial, guidelines are provided for papers where tools for pattern formation are central to the presented thesis, including situations where the details of the patterning tool configuration are important for understanding the information presented in a paper.

As with prior guidelines, details do not need to be provided explicitly if they are available in cited references. It is also not essential to provide details on aspects of exposure tools that are not pertinent to the thesis of the paper. For example, a paper on a new illumination system need not include descriptions of alignment.

This current set of guidelines is broken into two separate groups: one covering discussions focused on the development of patterning equipment, and the other focused on work that utilizes patterning equipment for achievement of a novel result. It is quite possible that an article will contain aspects of both, where a tool hardware configuration is presented and demonstrated on wafers, in which it would be appropriate to address aspects of both lists presented below. First, we present the guidelines for hardware improvements:

\section{Patterning Tooling Guidelines}

- Detailed description of patterning tool capability

- Patterning scheme for which performance is evaluated

- How the tool differs from prior state-of-the-art

- For new models of existing tools, changes and the impact on overall tool performance should be provided.

- If changes are made to one part of an exposure tool, the impact on other aspects of performance should be described.

- Throughput

- Defectivity and cleanliness

- Reliability and failure modes

- Throughput

○ Conditions at which throughput is specified

- Exposure dose

- Usage or non-usage of pellicle (and transmission of pellicle)

- Size and number of exposure fields per wafer

- Minimal pupil fill, illumination, numerical aperture, wavelength(s)

- Number of alignment points measured

- Overlay performance, focus control, and critical dimension performance at the stated throughput

(C) 2021 Society of Photo-Optical Instrumentation Engineers (SPIE) 
- Usage of any non-standard operations and their impact on throughput

- Description and impact of presented hardware, configuration, or process changes

- Example: With or without reticle/template exchange (e.g., use of multiple reticles)

- Required calibrations to maintain performance

- Measurement on wafer vs. integrated component (e.g., wavefront/dose sensor)

- Frequency and length of time during which wafers cannot be patterned

- Data utilization for feedforward process correction and tool monitoring

- Alignment

- Mark layout and design, image or diffraction-based methodology, feedforward modeling and correction methodology, front/back substrate, wavelength(s) utilized

- Positioning metrology (interferometers, encoders, etc...)

- Wafer conditioning, such as thermalization

- Any that is required prior to placing wafers in exposure tools

- Conditioning within exposure tools that could detract from other metrics of interest, such as throughput

\section{Patterning Applications Guidelines}

- Description of lithographic methodology

- Means of patterning, including optical/EUV, nanoimprint lithography (NIL), interference, multi-color, electron beam, etc.

- For commercially available tools that are used

- Manufacturer

- Tool name

- Series/model number

- Impact of the lithography process on exposure tool productivity

- Lithographic performance

- Resolution

- $\mathrm{CDU}$

- LER/LWR

- Including description of methods used to address measurement noise

- Overlay

- Defectivity (or other yield detractors) achieved

- Patterning conditions

- Optical: source shape, polarization, exposure dose, wavelength(s), numerical aperture

- E-beam: total electron beam current, dose, landing voltage, number of beams, charge mitigation scheme, exposed area, write speed at the experimental conditions

- Nanoimprint: mechanical operation of the process, coating, cross-linking methods, alignment/overlay capabilities, models, and methods

- Interference: spatial coherence, temporal coherence, flux at source, flux on wafer, flux stability, image contrast, polarization, exposures dose, wavelength(s), number of beams, environment (vacuum, air, or gas) 
- Mask/template

- Mask/template impact on experimental results

- Mask-patterning co-optimization

- Imprint stamp lifetime (masters/replicas)

- Process flow

- Pre-patterning process stack (substrate, topography, planarization, anti-reflection, underlayer, and topcoat films)

- Patterning materials (resists, NIL materials)

- Additional materials (release film, adhesion layer, charge dissipation film, etc.)

- Pre- and post-patterning process flows: bakes, coats, rinses, wafer thermalization, pattern transfer methods

- Pattern fidelity improvement and smoothing methods (e.g., flood exposure)

- Alignment and overlay control methodology

- Number of alignment points measured, wavelength utilized, mark type and method

- Overlay feedforward modeling and correction scheme, inclusion of wafer topography

- Raw data to presented data conversions and compensation, including outlier removal

\section{Acknowledgments}

We would like to thank the following people for their contributions in the generation of these guidelines: Dr. Erik R. Hosler, PsiQuantum LLC; Dr. Timothy A. Brunner, ASML; Dr. Chris Progler, Photronics Inc.; Dr. Qinghuang Lin, Lam Research; and Dr. Mircea Dusa, imec. 